\title{
Modeling internal waves in a two-layer fluid flow
}

\author{
Gulmira Zhanbirova ${ }^{1}$, Galia Zavyalova ${ }^{1}$, Elena Muraveva $^{2}$, and Daryn Shabdirov,"* \\ ${ }^{1}$ Faculty of Information Technology, Atyrau Oil and Gas University, Atyrau, Kazakhstan \\ ${ }^{2}$ Department of Automated Technological and Information systems, Ufa State Petroleum Technological University, Ufa, Russian \\ Federation
}

\begin{abstract}
This study presents mathematical model of the internal waves and examines wave propagation in a two-layer fluid flow. Elements of the functional-analytical approach are used to develop the model. A flat unsteady motion of a two-layer liquid under a cover over a flat bottom is considered. The fluid is assumed to be ideal and incompressible. Internal waves are caused by external pressure application to the interface between the layers, oscillation of the flat bottom and disturbances in the flow. The power of the wave source is characterized by dimensionless parameter $\varepsilon$. The problem is formulated, and its solution is based on asymptotic analysis for $0<\varepsilon<1$.
\end{abstract}

\section{Introduction}

The mathematical formulation of internal waves is in particular interest for researchers as it has wide range of application in the industry, from oil transportation to deep sea constructions. More detailed review of the theory can be found in [1]. The main formulations are based on the full Euler system equations, which are fully nonlinear and computationally expensive to solve. More convenient and easier methods of describing the internal waves can be achieved by the use of asymptotic models with introduction of the dimensionless parameters. There are several works on a two-fluid systems, including studies based on the rigid-lid assumption [2, 3], weakly nonlinear models for the free-surface case [4], stronly nonlinear models $[5,6,7,8,9]$.

Fluid flow generated by an oscillating pressures, which move along the free surface with a constant velocity was previously studied in $[10,11,12]$. The problem of the motion of a dipole under a free boundary (the dipole oscillates with a constant frequency) was investigated in [13, 14]. In most works, a linear approximation was used, and it was reported that the linear approximation becomes invalid for some values of the used parameters. In $[11,12]$ nonlinear effects are taken into account and a uniformly suitable approximate solution is constructed.

In this paper, the motion of a two-layer fluid is studied using asymptotic analysis for the case where dimensionless property of the source power $\varepsilon$ is less than unity. The problem is formulated first and asymptotic expansion of its solution is developed when $\varepsilon$ approaches zero. The successive approximations are described by linear initial-boundary value problems; therefore, at each step, the solution can be found explicitly. The properties of this solution are investigated in relation to the input data of the problem.
A flat unsteady motion of a two-layer fluid under a cover over a flat bottom is considered. The following assumptions are adopted:

1) The fluid is ideal and incompressible ;

2) Surface tension is neglected ;

3) The fluid motion is uniform with a constant velocity of $\mathrm{V}^{\prime}$ until some initial moment $\mathrm{t}^{\prime}=0$;

4) The source of internal waves begins to act at $t^{\prime}=0$ and oscillates with a frequency of $\omega^{\prime}$;

5) The source power is characterized by a dimensionless parameter $\varepsilon>0$;

6) We consider the case where $\varepsilon<1$;

7) There are three sources of internal waves:

a) pressure $\varepsilon p^{\prime}$ is applied to the interface between the layers;

b) some section of the bottom (or lid) oscillates according to a given law;

c) a regular or singular potential of disturbances is superimposed on the flow.

6) The movement in each layer is nonturbulent.

The problem is formulated as determination of the fluid flow and shape of the interface between the layers at $t>0$ according to the given mechanism of wave generation.

\section{Mathematical model}

\subsection{Formulation of the problem}

For simplicity, we will consider the problem within a coordinate system in which the motion of the fluid at $\mathrm{t}^{\prime}<0$ is a uniform flow. The $\mathrm{x}^{\prime}$ axis is directed along the unperturbed interface of the layers upstream, and the $y^{\prime}$ axis is oriented opposite to the direction of the free fall acceleration $\vec{g}$. Then at $\mathrm{t}^{\prime} \leq 0$ the interface equation is $\mathrm{y}^{\prime}$ $=0$. The depth of the lower layer (with density of $\rho_{2}^{\prime}$ ) is

\footnotetext{
* Corresponding author: chdaryn@mail.ru
} 
$\mathrm{H}_{2}^{\prime}$, and the depth of the upper layer (with density $\rho^{\prime}{ }_{1}$ ) is $\mathrm{H}^{\prime}{ }_{1}$. We assume that the lower layer is denser that the upper layer : $\rho_{2}^{\prime} / \rho_{1}^{\prime}>1$.

In order to move to the dimensionless variables, we assign the value of $\mathrm{V}^{\prime 2} / \mathrm{g}^{\prime}$ for the length scale, the value of $\mathrm{V}^{\prime} / \mathrm{g}^{\prime}$ - for the time scale, the value of $\rho^{\prime}{ }_{1} \mathrm{~V}^{\prime 2}$ - for the pressure scale, and the value of $\rho^{\prime}{ }_{1}$ - for the density scale :

$$
\begin{gathered}
\mathrm{H}_{\mathrm{j}}=\mathrm{H}_{\mathrm{j}} \mathrm{g}^{\prime} / \mathrm{V}^{\prime 2}, \\
\lambda=\rho_{2}^{\prime} / \rho^{\prime}{ }_{1}, \\
\omega=\omega^{\prime} \mathrm{V}^{\prime} / \mathrm{g}^{\prime}
\end{gathered}
$$

where $\mathrm{j}=1$ for the upper layer and $\mathrm{j}=2$ for the lower layer.

First we start with the case when the waves are generated by some disturbance potential. The fluid is at at rest until the initial moment $\mathrm{t}^{\prime}=0$. At $\mathrm{t}^{\prime}=0$, the wave generation mechanism begins to operate, which moves in the positive direction of the $x$ axis with a constant velocity of $\mathrm{V}^{\prime}$ and oscillates at a given frequency. Within the coordinate system which moves together with the source of perturbations (this system coincides with the system chosen above), we obtain the problem of waves on the flow.

Now we need to find the velocity potential $\Phi_{j}$ for the $\mathrm{j}$-th layer in the following form :

$$
\varphi_{j}(x, y, t, \varepsilon)=-x+\varphi_{j}(x, y, t, \varepsilon)+\varepsilon \chi_{j}(x, y, t)
$$

where $\chi_{j}$ are the given perturbation potentials (possibly different for each layer), the parameter $\varepsilon$ characterizes the power of the source. For the functions $\varphi_{\mathrm{j}}$, we have the following initial-boundary value problem:

$$
\begin{gathered}
\varphi_{1}=0 \quad\left(\eta(x, t, \varepsilon)<y<H_{1}\right) \\
\Delta \varphi_{2}=0 \quad-H_{2}<y<(\eta(x, t, \varepsilon) \\
\left(\varphi_{1}-\varphi_{2}\right)_{y}=\eta_{x}\left(\varphi_{1}-\varphi_{2}\right)_{x}- \\
-\varepsilon\left(\chi_{1}-\chi_{2}\right)_{y}+\varepsilon \eta_{x}\left(\chi_{1}-\chi_{2}\right)_{x} \\
\left(\varphi_{1 t}-\varphi_{2 x}\right)+\eta-\lambda\left[\varphi_{2 t}-\varphi_{2 x}+\eta\right]=0,5 \lambda\left|V\left(\varphi_{2}-\varepsilon \chi_{2}\right)\right|^{2}- \\
-0,5\left|V\left(\varphi_{1}-\varepsilon \chi_{1}\right)\right|^{2}+\varepsilon \lambda\left(\chi_{2 t}-\chi_{2 x}\right)-\varepsilon \lambda\left(\chi_{1 t}-\chi_{1 x}\right) \\
\eta_{t}-\eta_{x}-\varphi_{2 y}=\varepsilon \chi_{2 y}-\eta_{x}\left(\varphi_{2}-\varepsilon \chi_{2}\right)_{x} \\
(y=(\eta(x, t, \varepsilon)) \\
\varphi_{1 y}=-\varepsilon \chi_{1 y} \quad\left(y=H_{1}\right) \\
\varphi_{2 y}=-\varepsilon \chi_{2 y} \quad\left(y=-H_{2}\right) \\
\varphi_{1}=\varphi_{2}=\eta=0 \quad(t \leq 0) \\
|\eta|<\infty,\left|V \varphi_{j}\right|<\infty(t>0)
\end{gathered}
$$

Here (2) and (3) are equations of motion in the corresponding regions, (4) and (5) are the continuity conditions of the normal component of the velocity and pressure vectors at the interface $\mathrm{y}=\eta(\mathrm{x}, \mathrm{t}, \varepsilon)$, respectively; (6) - kinematic condition, (7) and (8) are the conditions of impermeability on the lid and bottom, (9) is the initial condition. Eq. (10) implies that we consider the flows, the velocity field of which is described by bounded functions everywhere in the region occupied by the fluid.

If the internal waves are caused by the oscillating pressures $\varepsilon p$, then $\chi_{j} \equiv 0(j=1,2)$ in Eqs. (1) - (10) and Eq. (5) should be replaced by the following condition :

$$
\begin{aligned}
& \varphi_{1 t}-\varphi_{1 x}+\eta-\lambda\left[\varphi_{2 t}-\varphi_{2 x}+\eta\right]+ \\
& +0,5\left|V \varphi_{1}\right|^{2}-\lambda\left|V \varphi_{2}\right|^{2}=\varepsilon p(x, t)
\end{aligned}
$$

In the case of an oscillating deformation of the bottom or cover, we set $\chi_{j} \equiv 0$, and condition (7) is replaced by

$$
\Phi_{2 y}=\varepsilon\left(f_{t}+\varphi_{2 x} f_{x}\right) \quad\left(y=-H_{2}+\varepsilon f(x, t)\right)
$$

\subsection{Asymptotic solution}

The functions $\varphi_{\mathrm{j}}(\mathrm{x}, \mathrm{y}, \mathrm{t}, \varepsilon)$ and $\eta(\mathrm{x}, \mathrm{t}, \varepsilon)$ will be sought for $\varepsilon<1$ in the following form :

$$
\begin{aligned}
& \varphi_{i}=\varepsilon \varphi_{i}^{(0)}+\varepsilon^{2} \varphi_{i}^{(1)}+\mathrm{K} \quad(j=1,2) \\
& \eta=\varepsilon \eta^{(0)}+\varepsilon^{2} \eta^{(1)}+\ldots
\end{aligned}
$$

Combination of Eq. (11) and Eqs. (2) - (10) along with keeping the leading terms when $\varepsilon \rightarrow 0$, we obtain that the main term of the asymptotics of the solution when $\varepsilon \rightarrow 0$ is described by a linear boundary value problem, which is obtained after disregarding nonlinear terms in Eqs. (2) - (10).

Successive approximations problem can be constructed using the Stokes method [6]. In this method, the functions $\varphi_{1}{ }^{(k)}$ and $\varphi_{2}{ }^{(k)}$ can be found at the k-th step so that they are harmonic in the strips $0<\mathrm{y}<\mathrm{H}_{1},-\mathrm{H}_{2}$ $<y<0$, respectively. In addition, each term in Eqs. (4) (6) can be expanded into Taylor series in the vicinity of the unperturbed position of the interface between the layers $(\mathrm{y}=0)$. For instance:

$$
\begin{aligned}
& \varphi_{1 y}(x, \eta(x, t, \varepsilon), t, \varepsilon)=\varphi_{1 y}(x, 0, t, \varepsilon)+ \\
& +\left.\frac{\partial^{2} \varphi_{1}}{\partial y^{2}}\right|_{y=0} \eta(x, t, \varepsilon)+\left.\frac{1}{2} \frac{\partial^{3} \varphi_{1}}{\partial y^{3}}\right|_{y=0} \eta^{2}(x, t, \varepsilon)+\ldots
\end{aligned}
$$

Then Eq. (11) is inserted into the above series. Moreover, at each step, we obtain a linear inhomogeneous initial-boundary value problem in a fixed domain. General form of this problem can be formulated as follows :

$$
\begin{array}{cl}
\Delta u_{1}=0 & \left(0<y<H_{1}\right), \\
\Delta u_{2}=0 & \left(-H_{2}<y<0\right.
\end{array}
$$




$$
\begin{gathered}
\left(u_{1}-u_{2}\right)_{y}=f_{1}(x, t \quad(y=0), \\
u_{1 t}-u_{1 x}+h-\lambda\left(u_{2 t}-u_{2 x}+h\right)=f_{2}(x, t) \\
(y=0) \\
h_{t}-h_{x}-u_{2 y}=f_{3}(x, t) \quad(y=0), \\
u_{1 y}=f_{4}(x, t) \quad\left(y=H_{1}\right), \\
u_{2 y}=f_{5}(x, t) \quad\left(y=-H_{2}\right), \\
u_{1}=u_{2}=h=0 \quad(t<0), \\
\left|V u_{j}\right|<\infty, \quad|h|<\infty \quad(t>0),
\end{gathered}
$$

Here $u_{i}=\varphi_{i}^{\left({ }^{(n)}\right.}, \mathrm{h}=\eta^{(n)}, \mathrm{j}=1,2, \mathrm{n}=0,1, \ldots$.

The functions $f_{k}(x, t)$ for the $n$-th step are determined through the previous approximations $\varphi_{i}{ }^{(0)}, \varphi_{i}{ }^{(1)}, \ldots \varphi_{i}{ }^{(n-1)}$ in accordance with the Stokes procedure.

Remark 1. The Stokes procedure is applicable if at each step we analytically obtain functions $\varphi_{j}{ }^{\left({ }^{n}\right)}, \varphi_{j t}{ }^{(n)}$ in $x, y$ and functions $\eta^{(n)}, \eta_{t}^{(n)}$ - in $x$.

This may impose considerable restrictions on the type of acceptable disturbances.

Remark 2. In case the waves are generated from oscillating bottom or due to the disturbances superimposed on the flow, the Stokes method is applied. The form of the successive approximations is identical to Eqs. (12) - (20).

Solution of Eqs. (2) - (10) in the exact form can be difficult, so that we consider the limit problem of Eqs. (12) - (20) under fairly general assumptions on the functions $f_{k}(x, t)(\mathrm{k}=1,2, \ldots, 5)$.

We propose to define the solution of Eqs. (12) - (20) as generalized functions of slow growth in $\mathrm{x}$, continuously differentiable in $\mathrm{y}$ in the corresponding domains. This function can be written as follows:

$$
\begin{gathered}
u_{1} \in C^{1}\left(\left[0, H_{1}\right] ; J^{\prime}\left(R^{2}\right)\right), \\
u_{2} \in C^{1}\left(\left[-H_{2}, 0\right] ; J^{\prime}\left(R^{2}\right)\right), h \in J^{\prime}\left(R^{2}\right)
\end{gathered}
$$

\subsection{Linear approximation}

If we insert the following equations

$$
\begin{gathered}
f_{1}\left(\chi_{2}-\chi_{1}\right)_{y}, f_{2}=\lambda\left(\chi_{2 t}-\chi_{2 x}\right)-\left(\chi_{1 t}-\chi_{1 x}\right) \\
f_{3}=\chi_{2 y}(y=0), f_{4}=-\chi_{1 y}\left(y=H_{1}\right), f_{5}=-\chi_{2 y}\left(y=-H_{2}\right)
\end{gathered}
$$

into Eqs. (12) - (20), then the functions $u_{1}, u_{2}$, $\mathrm{h}$ will result the leading terms of the solution asymptotics of the Eqs. (2) - (10) when $\varepsilon \rightarrow 0$. In accordance with condition $\left(7^{\prime}\right)$, we accept $\mathrm{f}_{k} \equiv 0, \quad(\mathrm{k}=1,2,3,4), \mathrm{f}_{5}=$ $f_{t}(x, t)$, then we obtain the asymptotics of the solution to the problem of fluid motion caused by periodic bottom deformations, etc. Thus, relations (12) - (20) describe a linear approximation in the problem of internal waves in a flow of a two-layer fluid with an arbitrary mechanism of wave formation.
We assume that $f_{k}(x, t)$ have the following properties:

1) $f_{k}(x, t) \equiv 0, \mathrm{t}<0$;

2) $f_{k}\left(x, t+\frac{2 \pi}{\omega}\right)=f_{k}(x, t), \mathrm{t}>0$,

3) $\quad f_{k}(x, t)$ are absolutely integrable with respect to $\mathrm{x}$ for $-\infty<\mathrm{x}<+\infty$;

4) $f_{k}(x, t)$ are integrable with square in $t$ on the interval $(0,2 \pi / \omega)$;

5) The functions $\int_{-\infty}^{+\infty} \mathrm{fk}(\mathrm{x}, \mathrm{t}) e^{-i x \xi} d x$ are Hölder in $\xi$.

It is known that functions of this type can be represented in the form of Fourier series

$$
f_{k}(x, t)=\sum_{n=-\infty}^{\infty} f_{k n}(x) e^{-i \omega n t}
$$

where

$$
f_{k n}(x)=\frac{\omega}{2 \pi} \int_{0}^{2 \pi / \omega} f_{k}(x, t) e^{-i \omega n t} d t
$$

Since Eqs. (12) - (20) are linear, it is sufficient to know their solution only for a special form of the righthand sides

$$
f_{k}(x, t)=\sum_{n=-\infty}^{\infty} f_{k n}(x) e^{-i \omega n t}
$$

where $H(t)$ is the Heaviside function, $H(t)=0$ for $t \leq 0$ and $\mathrm{H}(\mathrm{t})-1$ for $\mathrm{t}>0$. In this case, the complete solution is built according to the superposition principle.

It is convenient to deal with the the velocity field rather than potentials $\mathrm{u}_{1}, \mathrm{u}_{2}$ :

$$
\mathrm{u}_{1 \mathrm{y}}(\mathrm{x}, \mathrm{y}, \mathrm{t})=\sum_{n=1}^{5} M_{n} f_{n} H(t)
$$

where operators $\mathrm{M}_{\mathrm{n}}$ act according to the rule

$$
M_{n} \mathrm{v}(\mathrm{x})=\frac{1}{2 \pi} \int_{-\infty}^{\infty} \mathrm{v}^{F} \mathrm{Kn}(\xi, y, t) e^{i \xi x} d \xi
$$

The symbols $K n(\xi, y, t)$ of these operators have the following form :

$$
\begin{aligned}
& K_{1}(\xi, y, t)=-q_{0}^{-1}(\xi)\left\{S(\xi, t)-\frac{\frac{\lambda}{\lambda+1}\left(1-\frac{t h \xi H 1}{t h \xi H 2}\right) e^{\text {iet } r h \xi(y-H 1)}}{\operatorname{sh\xi } \xi 1}\right\} \\
& K_{2}(\xi, y, t)=\frac{1}{\lambda+1}\left\{(\omega-\xi) S(\xi, t)+\frac{\frac{1}{2} \pi(\xi)\left(e^{i \pi 2 t}-e^{i \pi t t}\right) s h \xi(y-H 1)}{s h \xi H 1}\right\}(24) \\
& K_{3}(\xi, y, t)=S(\xi, t) \frac{s h \xi(y-H 1)}{\operatorname{sh} \xi H 1} \\
& K_{4}(\xi, y, t)=\left(q_{0}(\xi) \operatorname{ch} \xi H_{1}\right)^{-1}\left\{\varepsilon(\xi, t)-e^{i \omega x}\right\} \frac{\operatorname{sh} \xi(y-H 1)}{\operatorname{sh} \xi H 1}+\frac{\operatorname{sh} \xi y e^{i o t}}{\operatorname{sh} \xi H 1} \\
& K_{5}(\xi, y, t)=\lambda \frac{s h \xi H 1}{s h \xi H 2}\left(q_{0}(\xi) \operatorname{ch} \xi H_{1}\right)^{-1}\left\{S(\xi, t)-e^{i e r \gamma}\right\} \frac{s h \xi(y-H 1)}{s h \xi H 1}
\end{aligned}
$$

where $\mathrm{q}_{0}(\xi)=\mathrm{q}^{\prime}(\xi)$ th $\xi \mathrm{H}_{1}, \quad \pi_{1}(\xi)=\xi+\pi(\xi), \quad \pi_{2}(\xi)=\xi-\pi(\xi)$, $\pi(\xi)=\sqrt{(\lambda-1) \xi /\left(q^{`}(\xi)\right)}, q^{\prime}(\xi)=\lambda \operatorname{cth} \xi \mathrm{H}_{2}+\operatorname{cth} \xi \mathrm{H}_{1}$, $\mathrm{S}(\xi, \mathrm{t})=\frac{\pi(\xi)}{2}\left\{\frac{e^{i \pi 2 t}-e^{i \omega t}}{\pi 2-\omega}-\frac{e^{i \pi 1 t}-e^{i \omega t}}{\pi 1-\omega}\right\}$. 
Similarly, Fourier series can be constructed for $\mathrm{u}_{2 \mathrm{y}}(\mathrm{x}, \mathrm{y}, \mathrm{t}), \mathrm{h}(\mathrm{x}, \mathrm{t})$.

Properties of $K_{j}(\xi, y, t)$ can be listed as the following :

a) infinitely many times differentiable with respect to $\mathrm{y}, \mathrm{t}$ for any $\xi$;

b) continuous in $\xi$;

c) infinitely differentiable with respect to $\xi$ for $\xi \neq 0$;

d) for $0<y<H_{1}$ decreases (together with its derivatives with respect to $y$ and of any order) as $|\xi| \rightarrow$ $\infty$ faster than any degree $|\xi|^{-1}$;

e) $\mathrm{K}_{1}=0\left(|\xi|^{-1 / 2}\right), \mathrm{K}_{2}=0(1), \mathrm{K}_{3}=0\left(|\xi|^{-1 / 2}\right), \mathrm{K}_{4}=0\left(e^{-|\xi| H 1}\right)$ $\mathrm{K}_{5}=0\left(e^{-|\xi| H 2}\right)$ for $\mathrm{y}=0,|\xi| \rightarrow \infty$.

The derivatives $K_{j \xi}$ have a discontinuity of the first kind at $\xi=0$. From this we obtain that for $0<\mathrm{y} \leq \mathrm{H}_{1}$ and finite, the function has the following properties:

1) $u_{1 y} \in C^{\infty}\left(R^{1} x\left(0, H_{1}\right) x(0, \infty)\right)$;

2) $\quad \mathrm{u}_{1 \mathrm{y}}=0(1)$ as $|\mathrm{x}| \rightarrow \infty$;

3) $\mathrm{u}_{1 \mathrm{y}}=\mathrm{f}_{4}(\mathrm{x}) e^{i \omega t}$ for $\mathrm{y}=\mathrm{H}_{1}, \mathrm{t}>0$.

The behavior of $u_{1 y}$ when $|x| \rightarrow \infty$ can be found more precisely if we know properties of $\mathrm{f}_{\mathrm{k}}(\mathrm{x})$. So that, if not only $f_{k}(x)$, but also $\mathrm{xf}_{\mathrm{k}}(\mathrm{x})$ is absolutely integrable function (in this case, $\mathrm{f}_{\mathrm{k}}^{\mathrm{F}}(\mathrm{x}) \in C^{1}(\mathrm{R})$, then $\mathrm{u}_{1 \mathrm{y}}=0\left(|\mathrm{x}|^{-1}\right)$ for $|\mathrm{x}| \rightarrow \infty$. Note, however, that if we require a higher smoothness of $\mathrm{f}_{\mathrm{k}} \mathrm{F}(\mathrm{x})$, then the rate of decrease of $\mathrm{u}_{1 \mathrm{y}}$ as $\mathrm{x} \mid \rightarrow \infty$ does not increase $\left(\mathrm{K}_{\mathrm{j} \xi}\right.$ are discontinuous functions for $\xi=0$ ).

The study of the properties of the operators $M_{n}$, defined by equality (23), for $y=0$ is nontrivial and is not carried out here. The above statements about the behavior of $\mathrm{u}_{1 \mathrm{y}}(\mathrm{x}, \mathrm{y}, \mathrm{t})$ are valid for finite $\mathrm{t}$ and become invalid as $\mathrm{t} \rightarrow \infty$. This fact can be demonstrated using a simple model example, which shows that the function $\mathrm{S}$ $(\xi, t)$ is not bounded as $t \rightarrow \infty$. Consider the function $F(x$, $\mathrm{a})=\left(1-e^{i a x}\right) / \mathrm{a}$. If $\mathrm{x}$ takes finite values, then $\mathrm{F}(\mathrm{x}, \mathrm{a})=$ $0(1)$ as $\mathrm{a} \rightarrow 0$. However, as $\mathrm{a} \rightarrow 0$ and $\mathrm{x}=0\left(\mathrm{a}^{-1}\right)$, the function $F(x, a)$ has order $0\left(a^{-1}\right)$, that is, it is not bounded as a $\rightarrow 0$.

In order to study the behavior of $\mathrm{u}_{1 \mathrm{y}}(\mathrm{x}, \mathrm{y}, \mathrm{t})$ at large $\mathrm{t}$, it is convenient to represent the symbols $\mathrm{K}_{\mathrm{j}}(\xi, \mathrm{y}, \mathrm{t})$ in the form $(j=1, \ldots, 5)$

$$
\begin{aligned}
& \mathrm{K}_{\mathrm{j}}(\xi, \mathrm{y}, \mathrm{t})=\mathrm{k}_{\mathrm{j} 0}(\xi, \mathrm{y})\left(\frac{e^{i \pi 2 t}}{\pi 2-\omega}-\frac{e^{i \pi 1 t}}{\pi 1-\omega}-\frac{e^{i \omega t}}{\pi 2-\omega}+\right. \\
& +\frac{e^{i \omega t}}{\pi 1-\omega}+\mathrm{k}_{\mathrm{j} 1}(\xi, \mathrm{y})\left\{e^{i \pi 2 t}-e^{i \pi 1 t}+\mathrm{k}_{\mathrm{j} 2}(\xi, \mathrm{y}) e^{i \omega t}\right.
\end{aligned}
$$

the functions $\mathrm{k}_{\mathrm{jn}}(\xi, \mathrm{y})(\mathrm{j}=0,1,2)$ are easy to find by comparing (24), (25).

The values of $\xi$ for which $\pi_{1}(\xi)=\omega$ or $\pi_{2}(\xi)=\omega$ are singular for $\mathrm{K}_{\mathrm{j}}(\xi, \mathrm{y}, \mathrm{t})$. The equation $\pi_{1}(\xi)=\omega$ has two negative roots $\xi_{1}, \xi_{2}\left(\xi_{1}<\xi_{2}<0\right)$ and one positive $\xi_{3}>0$ root for sufficiently small $\omega$ and under the condition $\pi$ ' $(+0)>$ 1 . With increasing $\omega$, the difference $\xi_{2}-\xi_{1}$ decreases and at some $\omega_{0}$ it vanishes (the roots "merge"). It is clear that $\omega_{0}=\pi\left(\xi_{0}\right)$, where $\xi_{0}$ is such that $\pi^{\prime}\left(\xi_{0}\right)=-1$. The equation $\pi_{2}(\xi)=\omega$ has for all $\omega>0$ a unique positive root $\xi_{4}$.

According to [10], we obtain the asymptotics of $\varphi_{\mathrm{y}}{ }^{(0)}(\mathrm{x}, \mathrm{y}, \mathrm{t})$ is unbounded when $\mathrm{t} \rightarrow \infty$ in the domain $\{\mathrm{x}$, $\left.\mathrm{y}|| \mathrm{x} \mid<<\mathrm{t}, 0<\mathrm{y}<\mathrm{H}_{1}\right\}$ only if there is a natural number $\mathrm{N}_{0}$ such that: a) $\omega_{0}$ and $N_{0} \omega$ are not separated, so it is impossible to specify disjoint neighborhoods of these points independent of $\varepsilon, \mathrm{t}$;

b) $\mathrm{f}_{\mathrm{kN} 0}(\mathrm{x}) \neq 0$ for at least one $\mathrm{k}(1 \leq \mathrm{k} \leq 5)$.

Thus, the resonance phenomenon is associated not only with the oscillation frequency of the wave source, but also with the set of harmonics (21) that describe these oscillations. In this case, the behavior of $\varphi_{y(0)}(x, y, t)$ at large values is determined by the following relation :

$$
\begin{aligned}
& \varphi_{y^{(0)}}(x, y, t)=\frac{A}{2} \delta^{-1 / 2} W_{0}\left(\xi_{0}, y\right) e^{i(\omega 0 t+\xi 0 x)} e^{-i t \delta} . . \\
& {\left[\sin \left(|x| A \delta^{1 / 2}\right)+\frac{1}{\pi} \sqrt{t \delta} \mathrm{G}(\mu, \varkappa) e^{-\frac{i A x \delta 1}{2}}\right]+0(1)} \\
& (t \rightarrow \infty, \delta \rightarrow 0) .
\end{aligned}
$$

here

$$
\mathrm{A}=\left(\frac{2}{\left|\pi^{\prime \prime}(\xi 0)\right|}\right)^{1 / 2} \quad, \delta=\omega_{0}-\mathrm{N}_{0} \omega, \quad \mu=-2 \sqrt{t \delta}-\mathrm{Ax} / \mathrm{t}^{1 / 2},
$$
$\varkappa=2 \sqrt{t \delta}, \quad W_{0}\left(\xi_{0}, y\right)=\sum_{j=1}^{5} f_{j N_{0}}^{F}\left(\xi_{0}\right) k_{j 0}\left(\xi_{0}, y\right)$, $\mathrm{G}(\mu, \mathcal{x})=\int_{-\infty}^{\infty} e^{-i(\xi 2+\mu \xi)} \frac{d \xi}{\xi(\xi-\varkappa)}$.

In the particular case of $\delta=0$ we have :

$$
\varphi_{y^{(0)}}(x, y, t)=\mathrm{t}^{1 / 2} \mathrm{~F}\left(\left(\mathrm{Ax} / \mathrm{t}^{1 / 2}, \mathrm{y}\right)+0(1) \quad(\mathrm{t} \rightarrow \infty)\right.
$$

where $F(\alpha, \beta)$ is a bounded function.

Remark 3. Eqs. (24) - (26) show that the form of the leading term of the asymptotic solution of the original problem when $\varepsilon \rightarrow 0, \delta \rightarrow 0, \mathrm{t} \rightarrow \infty$ does not depend on the specific implementation of the source of disturbances. Information on the mechanism of wave formation is contained in the constant $\mathrm{W}_{0}\left(\xi_{0}, 0\right)$, which determines the scale of the amplitude of asymptotics (26).

Remark 4. The possibility of resonance is determined not only by the nature of the formation of waves, but also by the internal properties of the system (the ratios $\left.\mathrm{H}_{2}{ }^{\prime} / \mathrm{H}^{\prime}{ }_{1}, \rho^{\prime}{ }_{2} / \rho^{\prime}{ }_{1}\right)$.

Indeed, for $\pi{ }^{\prime}(+0)<1$ the equation $\pi^{\prime}\left(\xi_{0}\right)=-1$ has no roots, thus for all $\omega>0$, the solution to the linear problem is bounded at $0 \leq \mathrm{t} \leq \infty$. Resonance occurs only at $\pi^{\prime}(+0)>1$. This condition can be described as :

$$
\mathrm{V}^{\prime}<\mathrm{v}_{0} \sqrt{H 2^{\prime}\left|g^{\prime}\right|}, \mathrm{v}_{0}=\left\{(\lambda-1) /\left(\lambda+\mathrm{H}_{2} / \mathrm{H}_{1}\right)\right\}^{1 / 2} .
$$

For example, if the top layer is absent $(\lambda=+\infty)$, then $\mathrm{v}_{0}=1$. Thus, only for sufficiently low flow velocities (motion of the wave source, respectively), one can expect the occurrence of resonance.

The constructed solution satisfies relations (2) - (10) with accuracy $0(\varepsilon)$ everywhere except for the region defined by the conditions $|\mathrm{x}| / \mathrm{t}<<1,\left|\mathrm{~N}_{0} \omega-\omega_{0}\right|<<1, \mathrm{t}$ $>$ 1. Inside this region, the linear approximation certainly loses its strength, and it is necessary to construct an additional expansion that describes the fine structure of the flow as $t \rightarrow \infty$. 


\section{Conclusion}

As a result of this study, mathematical model of the internal waves in a two-layer fluid flow was developed. The main outcomes can be summarized as follows :

a) The Stokes procedure is applicable if at each step we analytically obtain functions $\varphi_{j}{ }^{(n)}, \varphi_{j t}{ }^{(n)}$ in $x, y$ and functions $\eta^{(n)}, \eta_{t}^{(n)}-$ in $x$.

b) In case the waves are generated from oscillating bottom or due to the disturbances superimposed on the flow, the Stokes method is applied. The form of the successive approximations is identical to the case with applied pressures.

c) The form of the leading term of the asymptotic solution of the original problem when $\varepsilon \rightarrow 0, \delta \rightarrow 0, \mathrm{t} \rightarrow$ $\infty$ does not depend on the specific implementation of the source of disturbances. Information on the mechanism of wave formation is contained in the constant W0 $(\xi 0,0)$, which determines the scale of the amplitude of asymptotics.

d) The possibility of resonance is determined not only by the nature of the formation of waves, but also by the internal properties of the system (the ratios $\mathrm{H} 2$ '/H' 1 , $\left.\rho^{\prime} 2 / \rho^{\prime} 1\right)$.

The developed model satisfies the initial-boundary value problems with accuracy $0(\varepsilon)$ everywhere except for the region defined by the conditions $|\mathrm{x}| / \mathrm{t}<<1$, $\mathrm{N}_{0} \omega-\omega_{0} \mid<<1, \mathrm{t}>>1$. Inside this region, the linear approximation certainly loses its strength, and it is necessary to construct an additional expansion that describes the fine structure of the flow as $t \rightarrow \infty$.

\section{References}

1. K.R. Helfrich, W.K. Melville, Long nonlinear internal waves, Annu. Rev. Fluid Mech., Annual Reviews, Palo Alto, CA, 38, 395-425 (2006)

2. M. Miyata, An internal solitary wave of large amplitude, La mer, 23, 43-48 (1985)

3. Zh.L. Mal tseva, Unsteady long waves in a twolayer fluid, Dinamika Sploshn. Sredy, 193, 96110 (1989)

4. W. Choi, R. Camassa, Weakly nonlinear internal waves in a two-fluid system, J. Fluid Mech., 313, 83-103 (2006)

5. Y. Matsuno, A unified theory of nonlinear wave propagation in two-layer fluid systems, J. Phys. Soc. Japan, 62, 1902-1916 (1993)

6. R. Barros, S.L. Gavrilyuk, V.M. Teshukov, Dispersive nonlinear waves in two-layer flows with free surface, I. Model derivation and general properties, Stud. Appl. Math., 119, 191-211 (2007)

7. W. Choi, R. Camassa, Fully nonlinear internal waves in a two-fluid system, J. Fluid Mech., 396, 1-36 (1999)

8. A.E. Green, P.M. Naghdi, A derivation of equations for wave propagation in water of variable depth, J. Fluid Mech., 78, 237-246 (1976)
9. W. Craig, C. Sulem, P.-L. Sulem, Nonlinear modulation of gravity waves: A rigorous approach, Nonlinearity, 5, 497-522 (1992)

10. L. Debnath, S. Rosenblat, The ultimate approach to the steady state in the generation of waves on a running stream, Quart. Journ. Mech. and Appl. Math., 22 (2), 221-233 (1969)

11. T. Akylas, On the excitation of nonlinear water waves by a moving pressure distribution oscillating at resonant frequency, Phys. Fluids, 27 (12), 2803-2807 (1984)

12. G. Dagan, T. Miloh, Free-surface flow past oscillating singularities at resonant frequency, J. Fluid Mech., 120, 139-154 (1982)

13. I. Sturova, Generation of internal waves in a stratified fluid, In the book: Nonlinear problems of the theory of surface and internal waves (Novosibirsk: Science, 200-242, 1985)

14. H. Haussling, R. Coleman, Finite-difference computations using boundary-fitted coordinates for free-surface potential flows generated by submerged bodies, Proc. 2nd Intern. Conf. on Numerical Ship Hydrodynamics, Berkeley: Univ. of California, 221-233 (1977)

15. L. Sretensky, The theory of wave motion of fluid (M.: Nauka, 815, 1977)

16. A.A. Korobkin, Linear approximation in the problem of internal waves on a two-layer fluid flow, In the book: Problems of fluid dynamics with free boundaries (Novosibirsk: Dynamics of a continuous medium, 78-89, 1987) 